\title{
Acute effects of the Prevent injury and Enhance Performance warm-up on potential reinjury risk factors associated with unanticipated jump landings: Study protocol for a crossover randomized controlled trial in individuals cleared for return to sports after anterior cruciate ligament reconstruction.
}

Evans Y. K. Ashigbi ( $\nabla$ ekyayra@uhas.edu.gh )

Gollis University Faculty of Medicine https://orcid.org/0000-0003-3458-7850

Florian Giesche

Goethe-Universitat Frankfurt am Main

Winfried Banzer

Goethe-Universitat Frankfurt am Main

David A. Groneberg

Goethe-Universitat Frankfurt am Main

Daniel Niederer

Goethe-Universitat Frankfurt am Main

Study protocol

Keywords: Anterior Cruciate Ligament, Reinjury risk, Prevention, Return to sports, Jump landing quality, Performance, Unaticipated jump landing, Biomechanical landing safety, Neuromuscular, Warm-up

Posted Date: August 25th, 2020

DOI: https://doi.org/10.21203/rs.3.rs-33649/v1

License: (c) (i) This work is licensed under a Creative Commons Attribution 4.0 International License.

Read Full License 


\section{Abstract}

\section{Background}

In team-sports such as football or basketball, athletes need to rapidly adapt their motor plans and actions to unanticipated changes in the environment. Unanticipated jump-landing tasks have been found to elevate the risk of non-contact anterior cruciate ligament (ACL) injuries compared to an anticipated condition. ACL-reconstructed individuals may have greater difficulties to maintain neuromuscular control under unanticipated conditions exposing them to a higher reinjury risk during the game. The planned trial aims to investigate the acute effects of a team-sport specific injury prevention programme on potential ACL reinjury risk factors under anticipated and unanticipated jump-landings.

Methods and design:

Single center randomized controlled crossover trial. Female and male ACL-reconstructed participants cleared for return to sports ( $\geq 6$ months and $\leq 24$ months post-reconstruction) will be included. In a randomized sequence and with a washout phase of one week in between, the participants will perform an injury preventive warm-up protocol (PEP; strengthening, flexibility, plyometry and agility) and a standard warm-up program (bicycle ergometer). Both interventions will last for 12 minutes and will be conducted at moderate intensity (BORG scale: 12 to 14). After each warm-up, participants will perform counter movement jumps with single-leg landings on a force plate. Prior to the jump, a left or right footprint (equally distributed) will be indicated on a screen. Under the anticipated condition, the participants will be informed before the jump that the displayed footprint will not change after take-off. Under the unanticipated condition, the participants will not know whether the target landing side will remain the same (consistent with pre-movement expectations) or change (inconsistent). Under both unanticipated conditions, this information will be displayed 0.1 second after take-off and thus approximately 0.3 seconds before landing.

Parametric/non-parametric crossover-analyses (carryover-tests and crossover test) for betweenconditions comparisons will be applied.

Trial registration: German Clinical Trials Register, identification number DRKS00016942. Registered on May 24, 2019.

\section{Background}

Despite excessive pre- and postoperative neuromuscular rehabilitation, the risk for a second anterior cruciate ligament $(\mathrm{ACL})$ injury increases at least 10-folds when compared to the primary incidence.[1, 2] This risk is at its highest within the first two years after return to competitive sport.[3] It is therefore important to shift focus from rehabilitation to secondary/reinjury prevention after a certain period of postsurgical rehabilitation when the set return to sports (RTS) criteria are fulfilled. This may help address the persistent challenges posed by modifiable reinjury risk factors [4]through preventive training and 
performance enhancing approaches.[5-7] Since primary ACL injury prevention training programmes have been successful at reducing the risk of ACL injuries.[8] The transfer of such primary programmes to secondary injury prevention may be promising.

The use of preventive strategies with a focus on mechanisms of ACL graft rupture mostly include warmup exercises with dynamic single-leg jump landing manoeuvres, plyometrics, agility and neuromuscular strength enhancing activities.[9-11] Beyond strength and hop tests having predictive value for reinjury after ACL reconstruction (ACLR),[12] fast motor reaction/decision-making to unpredictable external signals may be another important reinjury risk predictive factor. During sports participation, athletes are required to rapidly process a multitude of visual stimuli (e.g. location and movement of ball, teammates and opponents) while simultaneously monitoring and adjusting their own motor plans and actions to unforeseen demands from the environment.[13, 14] Due to time constraints, decision-making within an extremely short time period during athletic movements (e.g. landing from a jump) does not often allow for sufficient feed-forward muscle contraction.[4, 15, 16] Accordingly, Almonroeder et. al.[17] found that these unanticipated affordances result in reduced biomechanical landing safety compared to anticipated tasks, where the stimulus indicating movement direction is shown prior to movement thereby increasing the odds of sustaining a non-contact ACL injury. In addition, unanticipated trials have been found to predispose athletes to wrong and prolonged landing-related decision-making (e.g. landing on the wrong side).[18-20] According to a previous study,[20] these decreases of biomechanical landing safety and decision-making quality under the unanticipated relative to anticipated can be defined as 'unanticipated landing costs'. These unanticipated landing costs may contribute to an increase in the residual risk for reinjury following RTS. Based on the reported high $A C L$ reinjury rates, $[2,3]$ one may speculate that ACLrecostructed individuals have greater difficulties in dealing with unanticipated circumstances.

In fact, Clark et. al.[21] found that ACL-reconstructed individuals exhibit altered landing mechanics during drop-jumps with unanticipated side-cuttings compared to an uninjured control group. Niederer et. al. [22], found that ACL-reconstructed patients show impairments in unanticipated jump landing movements for at least 18 to 26 months after surgery. This time span fits the proposed high-risk-for-a-second-ACL-injuryperiod[2, 3] after ACLR and RTS. Niemeyer et. al.[23] also reported that performing unanticipated single leg jump landing tasks following RTS clearance after ACLR provides additional information about functional restoration than classic RTS related hop-tests. These deficits and additional information areinvisible during anticipated landings since it is characterised by sufficient time for feed-forward control. The unanticipated conditions seem to reveal potentially persistent postoperative neuromuscular deficits leading to an increased reinjury risk on RTS.

Since, most injuries occur during a match or other competitive situation requiring time constrained decision making ACL-reconstructed athletes cleared to RTS may benefit in particular from the acute effects of injury preventive protocols aiming to improve neuromuscular performance. A promising neuromuscular preventive warm-up programme which may be used as a secondary preventive approach for ACL reinjury may be the Prevent Injury and Enhance Performance (PEP) programme. It was designed to target $A C L$ injury risk factors and is able to strongly reduce the incidence risk of primary ACL tears up 
to $82 \%$. [24]. As most of the preventive programmes, the PEP programme is utilized as a warm-up strategy. Several studies[4,25-28] investigated the acute effects of such warm-up programmes (in heathy participants) and a hypothetical positive impact on potential reinjury risk factors under both anticipated and unanticipated conditions in healthy individuals were found.

In ACL-reconstructed individuals, however, the potential acute effects of ACL-specific neuromuscular warm-up strategies on unanticipated jump landings ability are yet to be determined. Hence, the planned trial aims to determine the acute effects of the PEP warm-up protocol on potential (1) biomechanical reinjury risk factors during both anticipated/pre-planned and unanticipated/un-planned landing conditions as well as (2) unanticipated decision-making quality in ACL-reconstructed participants cleared for RTS. We hypothesise that an injury preventive warm-up programme (PEP) leads to improved jump landing stability and decision making quality in unanticipated relative to anticipated landings resulting in lower unanticipated landing costs.

\section{Methods And Design}

\section{Study design}

This study is a single centre crossover randomized control trial. It follows the Standard Protocol Items: Recommendations for Interventional Trials (SPIRIT) [29] and its accompanying explanation and elaboration [30] as depicted in the SPIRIT flow diagram in Fig. 1. The study was planned and being performed in accordance with the Helsinki declaration (Version Fortaleza Version). [31] The trial was prospectively registered in the German Clinical Trials Registry at: drks.de (registration number: DRKS00016942; 24. May, 2019).

\section{Ethics and informed consent}

Ethical approval was obtained from an independent Ethics Committee of the Goethe University, Frankfurt (Main), Germany (Faculty of Psychology and Sports Sciences). All prospective participants will be provided with two copies of written informed consent to sign and retain one after familiarising themselves with the relevant study participants' information.

\section{Sample size determination}

No study to retrieve the minimal clinical relevant change for the primary outcome during a single leg landing counter movemtn jump (CMJ) following an injury preventive warm-up programme on anticipated and unanticipated landing mechanics in ACL-reconstructed participants has been published yet. Therefore, our a priori sample size calculation (G*Power software version 3.1.9.2; Universität Düsseldorf) was based on standard assumptions. We considered a two-sided alpha-error (a) level of $5 \%$, a target effect size of 0.5 , and an $80 \%$ power. Accordingly, we will need data from 19 ACL-reconstructed participants (including an anticipated $10 \%$ drop out rate).

\section{Participants}




\section{Recruitment}

Participants' recruitment will be done primarily by contacting local surgeons and rehabilitations specialists, sports rehabilitation centres, physiotherapy and medical practices, sports clubs, fitness centres and the sports and medical campuses of the Goethe university in Frankfurt (Main), Germany by means of a poster with detachable contact information, flyers, telephone calls, e-mails as well as personal addressing. Additional recruitment will consist of advertisements on relevant professional and social media websites.

Inclusion criteria

Males and females engaged in level 1 or 2 sports (self-reported), thus $\geq 50$ hours/year;[32] prior to primary anterior cruciate ligament injury will be recruited. This study will involve adults aged 18 to 35 who have undergone primary, isolated and unilateral ACL-reconstruction ( $\geq 6$ and $\leq 24$ months after surgery). Included participants need to be cleared for return to sports from one's medical team (Physician and Physiotherapist).

\section{Exclusion criteria}

Participants will be excluded if they: (I) are not regular participants in level 1or 2 sporting activities (< 50 hours/year), (II) are < $6>24$ months after ACLR, (III) have a history of previous ACLR, (IV) have had 'unhappy Triad' in the knee joint (inner ligament, inner meniscus and cruciate ligament), (V) have muscle soreness, (VI) have pain in the lower extremity, (VII) have a history of other lower extremity injuries < 6 months requiring surgical repair, (VIII) have had a concussion < 6 months, (IX) currently take analgesics regularly/any other medication(s) that might influence the perception and physical performance, $(X)$ have any serious illnesses that is affecting their quality of life, (XI) are pregnant or breastfeeding.

Screening and inclusion

Only participants who meet the requisite inclusion criteria will be given an appointment for the test measurement by the research team. When a participant consent to be contacted by the research team, eligibility will be determined via e-mail or telephone conversation based on the inclusion and exclusion criteria for the study. Enrolled study participants will then receive all the preliminary study documents via e-mail to enable them adequately prepare for the appointment.

\section{Study flow and procedure}

All testing measures will be performed at the Goethe University Hospital, Frankfurt (Main), Germany, Division of Preventive and Sports Medicine.

Data assessment will take place at two separate days at comparable time of day with a washout phase of one week in between the toe days. At both days, all participants will familiarize themselves with the measurements. Participants will be guided to perform six test jumps ( 2 anticipated and 2 each for 
unanticipated 1 (consistent) and 2 (inconsistent) following a standardized mini warm-up (30 jumping jacks).

After a brief break, the intervention will be carried out. Then, all participants will perform the measurements. Primary and secondary outcomes will be assessed simultaneously. At the end, some participant characterizing variables and potential confounders (e.g. questionnaires and neuromuscular performance testing) will be collected. An overview of the study flow is provided in Fig. 1.

The measurements on both days are carried out under standardised conditions (e.g. light, temperature, test order, sportswear/shoes etc.).

\section{Preventive Warm-up protocols}

Two different warm ups; thus Prevent Injury and Enhance Performance (PEP) will be compared to a standard guideline Ergometer cycling of the same duration. All participants will undertake both the Prevent Injury and Enhance Performance (PEP) as well as the Ergometer cycling warm-up (see below) on two separated visits with a washout period of one week between measurements.

\section{Randomization procedure}

The order in which enrolled participants will perform the warm-up programmes will be randomized. The randomization list will determine whether a participant performs the PEP warm-up or ergometer cycling on test day 1 or 2. Microsoft Excel (Office 2016) software was used to generate 3 different randomization lists, one each for the starting warm-up condition, starting leg for single leg hop for distance and the order of the 3 different jump conditions (anticipated, unanticipated-consistent and unanticipated-consistent) to be tested.

\section{Prevent Injury and Enhance Performance (PEP): Intervention}

The PEP programme was developed by the Santa Monica Sports Medicine Foundation's ACL injury Prevention Project group as a strategic training programme to help reduce ACL injuries in female soccer players. It is a highly specific 15 -minute (10-minutes dynamic and 5-minutes stretching) training programme targeted at neuromuscular and proprioceptive problems identified in previous studies $[8,10$, 33]which have shown that neuromuscular and proprioceptive training can help reduce ACL injuries by two to four folds. This prevention program is subdivided into 5 sections (Section I: Warm-up, Section II: Strengthening, Section III: Plyometrics, Section IV: Agilities and Section V: Stretching) Since the focus of this trial is the biomechanical landing stability and decision making quality during a single leg CMJ only the dynamic component (Section I-IV): aiming to enhance neuromuscular performance and coordination of the lower extremity will be used. The warm-up programme will last for 12 minutes (including short breaks between exercises) and will be performed at a moderate intensity level (Borg Scale: 11-14). The participants will be asked after the first half of the programme to estimate their self-reported level of 
intensity. If the intensity exceeds or falls below the above-mentioned BORG range, participants will be informed to reduce or increase the tempo and the inter-exercise breaks, respectively. Immediately after the completion of the PEP programme, participants will be asked again to rate their subjective level of exhaustion before they will have a three to four minutes sitting rest $(B O R G \leq 10)$.

\section{Ergometer cycling: Control}

The participants will cycle (cadence of 70 to 80 repetitions per minute) on an electronic Ergometer (ergoline $\mathrm{GmbH}$, Bitz, Germany) at moderate intensity (Borg Scale 11-14) for 12 minutes just as the PEP intervention. The participants will be asked after two, six and eight minutes to estimate their subjective level of exhaustion. If the intensity exceeds or falls below the above-mentioned BORG range, the resistance (watt) will be adjust accordingly on an individual basis. Immediately after the completion of the cycling warm-up, participants will be asked again to rate their subjective level of exhaustion before they will have a three to four minutes sitting rest $(B O R G \leq 10)$ just as after PEP.

\section{Unanticipated and anticipated jump-landing assessments}

Immediately after each warm-up-programme, participants will perform repetitive CMJ (with hands placed at the hip) with single leg landings on a force plate; (HUMAC Balance System, Computer Sports Medicine Inc. Stoughton, Massachusetts, USA). A calibrated HUMAC Balance System was reported by Koltermann et. al. [34] to produce high quality and biomechanical measurement outcomes with minimal errors, similar to conventional force plates under static and dynamic conditions.

Three different conditions (randomly ordered) are to be completed: anticipated $(n=4)$, unanticipatedconsistent $(n=6)$ and unanticipated-inconsistent $(n=6)$ jump-landings (both sides equally distributed). Powerpoint slides (Microsoft Corporation, Redmond, Washington, USA) will be used to indicate the requested landing leg (left or right footprint relative to a vertical line). These slides will be shown on a 21.5-inch monitor placed at a height of $1.5 \mathrm{~m}$ and $2.5 \mathrm{~m}$ in front of the force plate. The slide depicting the requesting landing will be shown already before the jump. The take-off will lead to a release of a USB button foot switch (PC Sensor USB foot switch Keyboard; China) positioned under a plastic panel, where the participants are standing on prior to the jump. This button release will elicit a slide change with a latency of approximately 120 milliseconds (ms). Figure 2 illustrated the setup of the jump-landing task.

Regardless of the landing condition, participants will always start by standing on the plastic panel (with hands placed at the hip) while focusing the Powerpoint-slide being displayed on the screen in front of them. They are instructed to land on the side, which is illustrated before take-off (pre-defined plan/expectation) except the landing side change during the jump. After ground contact, the landing needs to be stabilized as fast as possible. The participants are asked to start the jump after a member of the research team instructed them verbally to do so.

Under the anticipated condition (Fig. 3), participants will be informed before take-off that the second Powerpoint-slide indicating the landing foot will be a repetition of the initial slide on the screen before 
take-off (i.e. no change of landing side after take). Therefore, the participants will have sufficient time to prepare their landing.

Under the unanticipated-consistent condition (Fig. 4), the landing side will not change during the jump as in the anticipated condition. That means that the landing information during the flight will be consistent with the landing side shown prior to the jump. In contrast to the anticipated condition, here, it is unknown to the participants during the preparation phase for the jump that the landing side will not change upon take-off. This condition will not require the participants to adapt their pre-defined motor plans during the jump.

Under the unanticipated-inconsistent condition (Fig. 5), the landing side illustrated before the jump will change to the other side after take-off (inconsistent with pre-movement expectation) without the participants knowing this before the jump. In contrast to the unanticipated-consistent trials, this condition will require the participants to adapt their pre-defined motor plans/expectations during the jump by switching to the other side. In both unanticipated conditions, participants will be asked to avoid speculation and guessing but to react to the stimulus appropriately.

1) Protective mat, 2) Hinge, 3) Plastic panel, 4) USB foot switch, 5) HUMAC balance force plate, 6) HDMI cable connecting laptop and USB foot switch to monitor, 7) Monitor/Screen (21.5 Inch), 8) Powerpointslide (projected) on screen prior to jump initiation (randomised)

A successful trial is defined as holding a stable landing position for at least 10 seconds (sec.). The participants will be allowed to use their arms to equilibrate the postural sway immediately after landing. After a stable standing position has been reached, their hands need to be repositioned on the hip while focusing the footprint on the monitor in front of them. Unsuccessful trials are categorised as standing errors (touching the ground with the free leg, leaving the force plate and touching the ground with the hands, and falls) or decision errors (landing on the wrong foot, landing on both foot). Previous studies $[35,36]$ revealed longer flight times for unanticipated jump-landings compared with anticipated landings. Therefore, participants will be encouraged to produce flight times of 400 to $500 \mathrm{~ms}$, which corresponds to a CMJ height of approximately 25 to $30 \mathrm{~cm}$ regardless of the condition. The corresponding available response times during the jump of 300 to 400 ms (flight times minus 120 ms latency of the button switch) are consistent with those of other studies [36, 37]. In order to achieve the required flight times, participants will practice the jump-landing task (anticipated: $n=4$, unanticipated-consistent/-inconsistent: $\mathrm{n}=4$ each, random ordered) during the familiarisation session at both days and will be provided with feedback regarding the achieved jump height after each jump. Those participants who are unable to reach a minimum flight time of $400 \mathrm{~ms}$ will be allowed to use their arms during take-off to gain momentum. If the required flight times are not reached during the actual jump-landing testing, participants will be informed accordingly and asked to consider that for the next trial. All participants will be expected to wear sports clothes (t-shirt and shorts) and indoor sports shoes during both task familiarisation session and the actual jump landing experiment. Participants will be encouraged to come along with their visual aids in case they use them during sports. 


\section{Primary outcome}

The vertical peak ground reaction force (pGRF) and the time to reach this maximum value (recorded with a sample frequency of $100 \mathrm{~Hz}$ ) upon landing will serve as the primary outcomes of this study. These values will be derived from the raw data of each trial.

\section{Secondary outcomes}

Postural landing stability: The centre of pressure (COP), time to stabilisation (TTS) and the number of standing errors will be used to operationalize the postural landing stability. Because a decreased dynamic postural stability has been found to be predictive for non-contact knee injuries in team sport athletes, the assessment of these variables is of particular relevance.

The COP path length reflects the cumulative postural sway (millimetre) of the center of mass recorded between the foot sole (area of support) and the force plate for a specific time.[38] The cumulative deviations of the vertical ground reaction forces will be calculated for the medio-lateral and anteriorposterior directions during the first 2.5 seconds after landing as this period corresponds to the early dynamic stabilization phase (Fransz et al. 2016).[39] The COP is considered a valid and reliable parameter for measuring postural control in different populations.[40] The TTS indicative for the time needed to regain a stable stance after landing relative to the whole stance time of ten seconds will be analysed in accordance with the specifications provided in Colby et. al.[41] Wikstrom et. al.[42] A moderate to high reliability has been demonstrated for the TTS in Jensen et. al.[43] .

\section{Decision making quality}

In order to operationalize the quality of decision making during the jump, the number of landing/decision errors (landing on the wrong leg or both legs) will be recorded and counted. In this context, "decisionmaking quality" refers to the ability to quickly adapt motor plans/intentions and actions to an unexpected visual landing information (landing on the left or right leg) during the jump. Standing errors will be defined as correct landings, which could not be stabilized over the ten seconds measuring period (i.e. ground contact of the free leg, leaving the force measuring plate, touching the ground/plate with the hands or fall).

The documentation of this type of error will be carried out for both the anticipated and the unanticipated conditions, although, this kind of error should only occur under the inconsistent unanticipated trials, because the other conditions do not require task switching. A high decision-making quality results in a lower number of decision errors and vice versa.

\section{Potential confounders}

\section{The following potential confounding variables will be assessed:}


- Single leg hop for distance (SLHD; maximum value of each side [cm] from three attempts). The SLHD is a valid and reliable test for assessing leg strength, neuromuscular control and dynamic knee stability in individuals after ACL reconstruction.[44] The reliability of this test is high with ICC of 0.97 (Cl 0.9-0.99) and a standard error measurement of 3.5\%.[45] A limb symmetry of 90 percent (jumping distance) is considered an essential prerequisite for the resumption of sports and competition activity after ACL reconstruction[44, 46, 47]

- Self-reported knee function of the ACL-reconstructed and uninjured knee using the Lysholm knee scale ( 0 = maximum restricted knee function; $100=$ no restricted knee function at all; Lysholm \& Gillquist, 1982; all test subjects). Good psychometric quality was demonstrated for the questionnaire. [48]

- Fear of re-injury associated with anticipated and unanticipated jump landings assessed by $10 \mathrm{~cm}$ visual analogue scale ( 0 = no fear at all, $10=$ maximum imaginable fear; all test persons) during the familiarisation session.

- Time since ACL-reconstruction (month).

- Mean flight time differential within and between anticipated and unanticipated jump-landing conditions (ms).

- Ability in selected cognitive functions (working memory and mental flexibility) found to be relevant to make fast and accurate landing-related decisions under time-constraints assessed via the trailmaking test version A and B [49] (time to test completion in seconds; higher values indicating lower test performance) after the jump-landing tasks on both days.

- Participants' current level of daily physical activity will also be evaluated using the the international physical activity questionnaire short form (IPAQ-SF). The validity (predictive, concurrent, convergent, criterion and discriminant) of this research tool is detailed in Lee et. al.,[50] with a high reliability of a $<0.80$. This questionnaire will be used to collect information from participants about time spent being physically active in the last 7 days.

\section{Statistics}

Parametric or non-parametric statistical tests will be selected after checking for all relevant underlying assumprions (data distribution, variance homogeneity and linearity).

All data will be reported descriptively as mean values, minima and maxima, standard deviations and 95\% confidence interval or, in the case of non-parametric analyses, in form of median and interquartile ranges.

The statistical analysis will be based on the intraindividually mean values/medians of the primary and secondary outcome measures. All outcome measures will first be tested for potential differences between the ACL-reconstructed and uninjured side.

Potential effects of both warm-up protocols will be evaluated for both sides separately (including limb symmetry) and non-side-specific (both limbs together). 
To identify potential carry-over effects between the two interventions, unpaired t-tests will be calculated to compare the intraindividually sums of both sequence groups (starting with PEP or cycling ergometer warm-up).

To investigate the implications of the assessed potential confounders on the between-condition differences explorative correlation analyses will be used. Potential significant between-condition flight time differences will be considered as covariates in the statistical analyses.

For the between-legs and between-warm-up programs' analyses, repeated measures analyses of variances ANOVAs (with the potential confounders as co-variates) or Friedman tests will be calculated.

In case of significant differences, Bonferroni-Holm adjusted post-hoc testing (paired t-tests/Wilcoxon tests) for the individual within and between-condition differences (unanticipated landing costs) will be applied.

The significance level (alpha error) will be set at $5 \%$ t. All statistical analyses will be performed using SPSS (IBM SPSS Statistics 24, Chicago, IL, USA) and Microsoft Excel 2016 (Microsoft Corporation, Redmond, Washington, USA). Effect sizes will be calculated using G*Power (Version: 3.1.9.2, University of Düsseldorf; Faul et al., 2007).

\section{Discussion}

The findings of the planned trial will help clinicians, therapists and athletic trainers to better estimate the suitability of a team-sport specific injury preventive warm-up programme to enhance acutely the ability of ACL-reconstructed athletes to deal with unanticipated circumstances on the pitch.

One major strength of our study is the fact that we will measure jump landing performance under unanticipated instead of just unplanned conditions as done in most of the related studies. This may better reflect the real playing situations where athletes do not know initially, if the circumstances will allow them to realize a pre-defined motor-plan (consistent with pre-movement expectation) or require them to adapt their motor-plans (inconsistent with pre-movement expectation) during a jump.

Furthermore, this distinction will enable us to identify those, who tend to guess or to just realize their initial expectations regarding the landing side without reacting to the stimulus during the jump (i.e. decision errors during consistent unanticipated trials), which was a limitation of a previous trial of our working group (Giesche et al. 2019). [35] Another strength relates to the fact, that we only considered physically active participants with a postoperative time within two years thereby increasing the homogeneity of our sample.

To sum up, the knowledge gained by our planned trial may provide novel insights into the implications of previous warm-up routines for potential secondary ACL-injury risk factors occurring under challenging athletic affordances. 


\section{Trial status}

The data collection for this study started in June, 2019 and the first participant was assessed on June 25, 2019. Recruitment is still ongoing at the time of submission of this manuscript. Final enrolment is planned to be completed by the end of August, 2020 and statistical analysis of the assessed data is expected to be completed by October, 2020. This is the first version of the protocol which was registered in the DRKS on May 24, 2019.

\section{Abbreviations}

ACL: Anterior cruciate ligament; ACLR: Anterior cruciate ligament reconstruction; RTS: Return to sports; PEP: Prevent injury and enhance performance; DRKS: German Clinical Trials Regisgry; SPIRIT: Standard protocol itmes: Recommendations for interventional trials; CMJ: Counter movement jump; pGRF: Peak ground reaction force; COP: Centre of pressure; TTS: Time to stabilization; SLHD: Single leg hop for distance; IPAQ-SF: Internation physical activity questionnaire-short form.

\section{Declarations}

\section{Availability of data and materials:}

Not applicable.

\section{Ethics approval and consent to participate:}

The study was approved from the local Ethics Committee of the Faculty of Psychology and Sport Science, Goethe-University Frankfurt (reference number: 2017/27). A written informed consent will be obtained from each participant. The data obtained will be anonymously kept and used only for the purspose of the study.

\section{Consent for publication:}

Not applicable

\section{Competing interests:}

None declared

\section{Funding:}

This study received no grant from any funding agency in the public, commercial or not-for-profit sectors. 


\section{Authors' contributions}

s: EYKA, FG and DN designed and put up the jump-landing setup, contributed to the development of the protocol and the first draft of the manuscript, revised the manuscript and provided final approval. WB and DAG revised the manuscript and provided intellectual contributions to the final, submitted version of the manuscript. All authors read and approved the final manuscript.

\section{Acknowledgements:}

Not applicable.

\section{Author details}

${ }^{1}$ Division of Preventive and Sports Medicine, Institute of Occupational, Social and

Environmental Medicine, Goethe University, Frankfurt am Main, Germany

${ }^{2}$ Department of Sports Medicine and Exercise Physiology, Institute of Sports Sciences, Goethe University, Frankfurt am Main, Germany

${ }^{3}$ Department of Physiotherapy and Rehabilitation Sciences, School of Allied Health Sciences, University of Health and Allied Sciences, Ghana

\section{References}

1. Niederer D, Engeroff T, Wilke J, et al. Return to play, performance, and career duration after anterior cruciate ligament rupture: A case-control study in the five biggest football nations in Europe. Scand J Med Sci Sports. 2018;28(10):2226-33.

2. Webster KE, Feller JA. Exploring the High Reinjury Rate in Younger Patients Undergoing Anterior Cruciate Ligament Reconstruction. Am J Sports Med. 2016;44(11):2827-32.

3. Wiggins AJ, Grandhi RK, Schneider DK, et al. Risk of Secondary Injury in Younger Athletes After Anterior Cruciate Ligament Reconstruction: A Systematic Review and Meta-analysis. Am J Sports Med. 2016;44(7):1861-76.

4. Niederer D, Willberg C, Kruse A, et al. Acute effects of preventive warm-up exercises on modifiable risk factors for anterior cruciate ligament injuries: a three-arm randomized-controlled crossover trial. $\mathrm{J}$ Sports Med Phys Fitness. 2020;60(1):92-101.

5. Fox AS. Change-of-Direction Biomechanics: Is What's Best for Anterior Cruciate Ligament Injury Prevention Also Best for Performance? Sports Med. 2018;48(8):1799-807.

6. Hewett TE, Ford KR, Myer GD. Anterior cruciate ligament injuries in female athletes: Part 2, a metaanalysis of neuromuscular interventions aimed at injury prevention. Am J Sports Med. 
2006;34(3):490-98.

7. Monajati A, Larumbe-Zabala E, Goss-Sampson M, et al. The Effectiveness of Injury Prevention Programs to Modify Risk Factors for Non-Contact Anterior Cruciate Ligament and Hamstring Injuries in Uninjured Team Sports Athletes: A Systematic Review. PLoS ONE. 2016;11(5):e0155272.

8. Taylor JB, Waxman JP, Richter SJ, et al. Evaluation of the effectiveness of anterior cruciate ligament injury prevention programme training components: a systematic review and meta-analysis. $\mathrm{Br} \mathrm{J}$ Sports Med. 2015;49(2):79-87.

9. Sugimoto D, Alentorn-Geli E, Mendiguchía J, et al. Biomechanical and neuromuscular characteristics of male athletes: implications for the development of anterior cruciate ligament injury prevention programs. Sports Med. 2015;45(6):809-22.

10. Hewett TE, Myer GD, Ford KR, et al. Mechanisms, prediction, and prevention of ACL injuries: Cut risk with three sharpened and validated tools. J Orthop Res. 2016;34(11):1843-55.

11. Myer GD, Ford KR, McLean SG, et al. The effects of plyometric versus dynamic stabilization and balance training on lower extremity biomechanics. Am J Sports Med. 2006;34(3):445-55.

12. Ashigbi EYK, Banzer W, Niederer D. Return to Sport Tests' Prognostic Value for Reinjury Risk after Anterior Cruciate Ligament Reconstruction: A Systematic Review. Med Sci Sports Exerc. 2020;52(6):1263-71.

13. Grooms DR, Onate JA. Neuroscience Application to Noncontact Anterior Cruciate Ligament Injury Prevention. Sports Health. 2016;8(2):149-52.

14. Swanik CB. Brains and Sprains: The Brain's Role in Noncontact Anterior Cruciate Ligament Injuries. J Athl Train. 2015;50(10):1100-02.

15. Whyte EF, Richter C, O'Connor S, et al. 2018 Investigation of the Effects of High- Intensity, Intermittent Exercise and Unanticipation on Trunk and Lower Limb Biomechanics During a Side-Cutting Maneuver Using Statistical Parametric Mapping. J Strength Cond Res. 2018;32(6):1583-93.

16. Krosshaug T, Nakamae A, Boden BP, et al. Mechanisms of anterior cruciate ligament injury in basketball: video analysis of 39 cases. Am J Sports Med. 2007;35(3):359-67.

17. Almonroeder TG, Garcia E, Kurt M. The effects of anticipation on the mechanics of the knee during single-leg cutting tasks: A Systematic Review. Int J Sports Phys Ther. 2015;10(7):918-28.

18. Mache MA, Hoffman MA, Hannigan $\mathrm{K}$, et al. Effects of decision making on landing mechanics as a function of task and sex. Clin Biomech (Bristol Avon). 2013;28(1):104-09.

19. Arumugam A, Markström JL, Häger CK. A novel test reliably captures hip and knee kinematics and kinetics during unanticipated/anticipated diagonal hops in individuals with anterior cruciate ligament reconstruction. J Biomech. 2020;99:109480.

20. Giesche F, Wilke J, Engeroff T, et al. Are biomechanical stability deficits during unplanned single-leg landings related to specific markers of cognitive function? J Sci Med Sport. 2020;23(1):82-8.

21. Clarke SB, Kenny IC, Harrison AJ. Dynamic knee joint mechanics after anterior cruciate ligament reconstruction. Med Sci Sports Exerc. 2015;47(1):120-27. 
22. Niederer $D$, Giesche $F$, Janko $M$, et al. Unanticipated jump-landing quality in patients with anterior cruciate ligament reconstruction: How long after the surgery and return to sport does the re-injury risk factor persist? Clin Biomech (Bristol Avon). 2020;72:195-201.

23. Niemeyer $P$, Niederer $D$, Giesche $F$, et al. Unanticipated jump-landing after anterior cruciate ligament reconstruction: Does unanticipated jump-landing testing deliver additional return to sport information to traditional jump performance tests? Clin Biomech (Bristol Avon). 2019;70:72-9.

24. Herman DC, Barth JT. Drop-Jump Landing Varies With Baseline Neurocognition: Implications for Anterior Cruciate Ligament Injury Risk and Prevention. Am J Sports Med. 2016;44(9):2347-53.

25. Bizzini M, Impellizzeri FM, Dvorak J, et al. Physiological and performance responses to the "FIFA 11+" (part 1): is it an appropriate warm-up? J Sports Sci. 2013;31(13):1481-90.

26. Ayala F, Calderón-López A, Delgado-Gosálbez JC, et al. Acute Effects of Three Neuromuscular WarmUp Strategies on Several Physical Performance Measures in Football Players. PLoS ONE. 2017;12(1):e0169660.

27. Salgado E, Ribeiro F, Oliveira J. Joint-position sense is altered by football pre-participation warm-up exercise and match induced fatigue. Knee. 2015;22(3):243-48.

28. Topcu H, Arabaci R. Acute effect of different warm up protocols on athlete's performance. E J Phys Edu Sports Sci. 2017;3(8):35-50.

29. Chan A-W, Tetzlaff JM, Altman DG, et al. SPIRIT 2013 statement: defining standard protocol items for clinical trials. Ann Intern Med. 2013;158(3):200-07.

30. Chan A-W, Tetzlaff JM, Gøtzsche PC, et al. SPIRIT 2013 explanation and elaboration: guidance for protocols of clinical trials. BMJ. 2013;346:e7586.

31. World Medical Association. Declaration of Helsinki: ethical principles for medical research involving human subjects. JAMA. 2013;310(20):2191-94.

32. Arundale AJH, Cummer K, Capin JJ, et al. Report of the Clinical and Functional Primary Outcomes in Men of the ACL-SPORTS Trial: Similar Outcomes in Men Receiving Secondary Prevention With and Without Perturbation Training 1 and 2 Years After ACL Reconstruction. Clin Orthop Relat Res. 2017;475(10):2523-34.

33. Rodríguez C, Echegoyen S, Aoyama T. The effects of "Prevent Injury and Enhance Performance Program" in a female soccer team. J Sports Med Phys Fitness. 2018;58(5):659-63.

34. Koltermann J, Gerber M, Beck H, et al. Validation of the HUMAC Balance System in Comparison with Conventional Force Plates. Technologies 2017;5(3):44.

35. Giesche F, Engeroff T, Wilke J, et al. Neurophysiological correlates of motor planning and movement initiation in ACL-reconstructed individuals: a case-control study. BMJ Open. 2018;8(9):e023048.

36. Brown TN, Palmieri-Smith RM, McLean SG. Sex and limb differences in hip and knee kinematics and kinetics during anticipated and unanticipated jump landings: implications for anterior cruciate ligament injury. Br J Sports Med. 2009;43(13):1049-56. 
37. Collins JD, Almonroeder TG, Ebersole KT, et al. The effects of fatigue and anticipation on the mechanics of the knee during cutting in female athletes. Clin Biomech (Bristol Avon). 2016;35:62-7.

38. Donath $L$, Roth $R$, Zahner $L$, et al. Testing single and double limb standing balance performance: comparison of COP path length evaluation between two devices. Gait Posture. 2012;36(3):439-43.

39. Fransz DP, Huurnink A, Boode VA de, et al. Time series of ground reaction forces following a single leg drop jump landing in elite youth soccer players consist of four distinct phases. Gait Posture. 2016;50:137-44.

40. Li Z, Liang Y-Y, Wang L, et al. 2016 Reliability and validity of centre of pressure measures for balance assessment in older adults. J Phys Ther Sci. 2016;28(4):1364-67.

41. Colby SM, Hintermeister RA, Torry MR, et al. Lower Limb Stability with ACL Impairment. The Journal of orthopaedic sports Physical Therapy. 1999;29(8):444-51. discussion 452-4.

42. Wikstrom EA, Tillman MD, Smith AN, et al. A new force-plate technology measure of dynamic postural stability: the dynamic postural stability index. J Athl Train. 2005;40(4):305-09.

43. Jensen RL. Reliability of time to stabilization in single leg standing. 27th Conference of the international society of Biomechanics in Sports 2009:346 - 49.

44. Logerstedt D, Grindem H, Lynch A, et al. Single-legged hop tests as predictors of self-reported knee function after anterior cruciate ligament reconstruction: the Delaware-Oslo ACL cohort study. Am J Sports Med. 2012;40(10):2348-56.

45. Kockum B, Heijne Al-LM. Hop performance and leg muscle power in athletes: Reliability of a test battery. Phys Ther Sport. 2015;16(3):222-27.

46. Kyritsis $P, B a h r ~ R$, Landreau P, et al. Likelihood of ACL graft rupture: not meeting six clinical discharge criteria before return to sport is associated with a four times greater risk of rupture. Br J Sports Med. 2016;50(15):946-51.

47. Grindem $H$, Snyder-Mackler L, Moksnes $H$, et al. Simple decision rules can reduce reinjury risk by $84 \%$ after ACL reconstruction: the Delaware-Oslo ACL cohort study. Br J Sports Med. 2016;50(13):804-08.

48. Kocher MS, Steadman JR, Briggs KK. et a 2004 Reliability, Validity, and Responsiveness of the Lysholm Knee Scale for various chondral disorders of the knee. J Bone Joint Surg Am. 2004;86(6):1139-45.

49. Sánchez-Cubillo I, Periáñez JA, Adrover-Roig D, et al. Construct validity of the Trail Making Test: role of task-switching, working memory, inhibition/interference control, and visuomotor abilities. J Int Neuropsychol Soc. 2009;15(3):438-50.

50. Lee PH, Macfarlane DJ, Lam TH, et al. Validity of the International Physical Activity Questionnaire Short Form (IPAQ-SF): a systematic review. Int J Behav Nutr Phys Act. 2011;8:115.

\section{Figures}




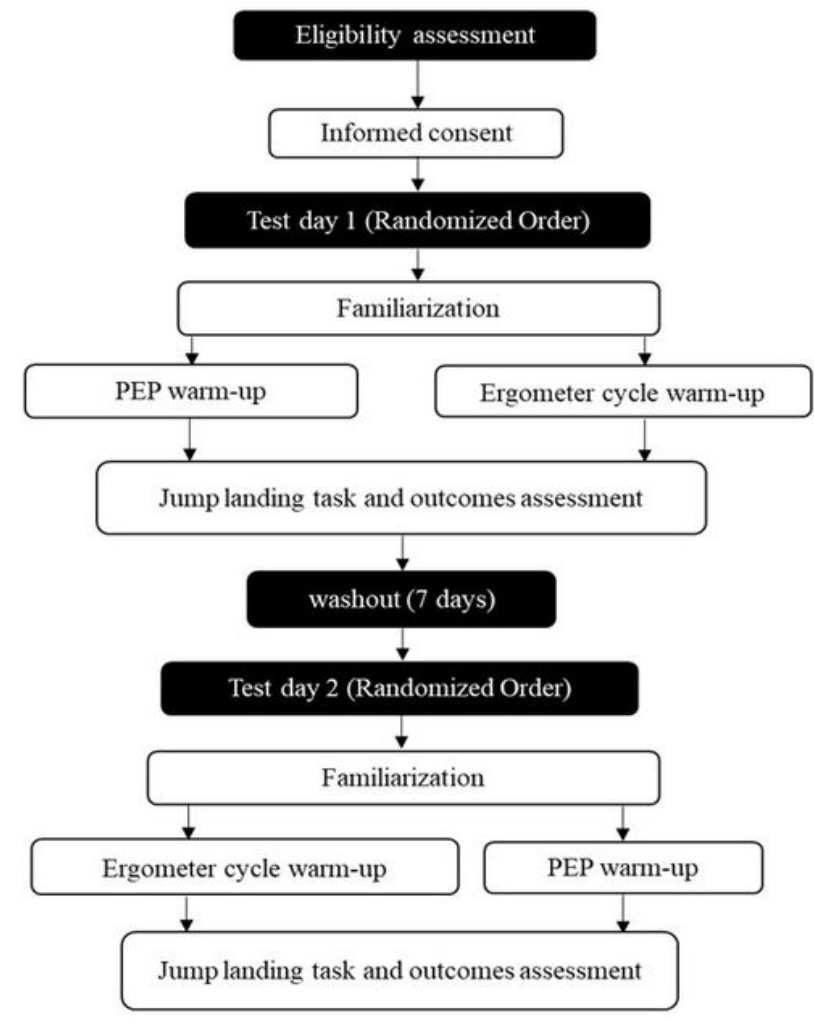

\section{Figure 1}

Standard Protocol Items: Recommendation for Interventional Trials (SPIRIT) flowchart.

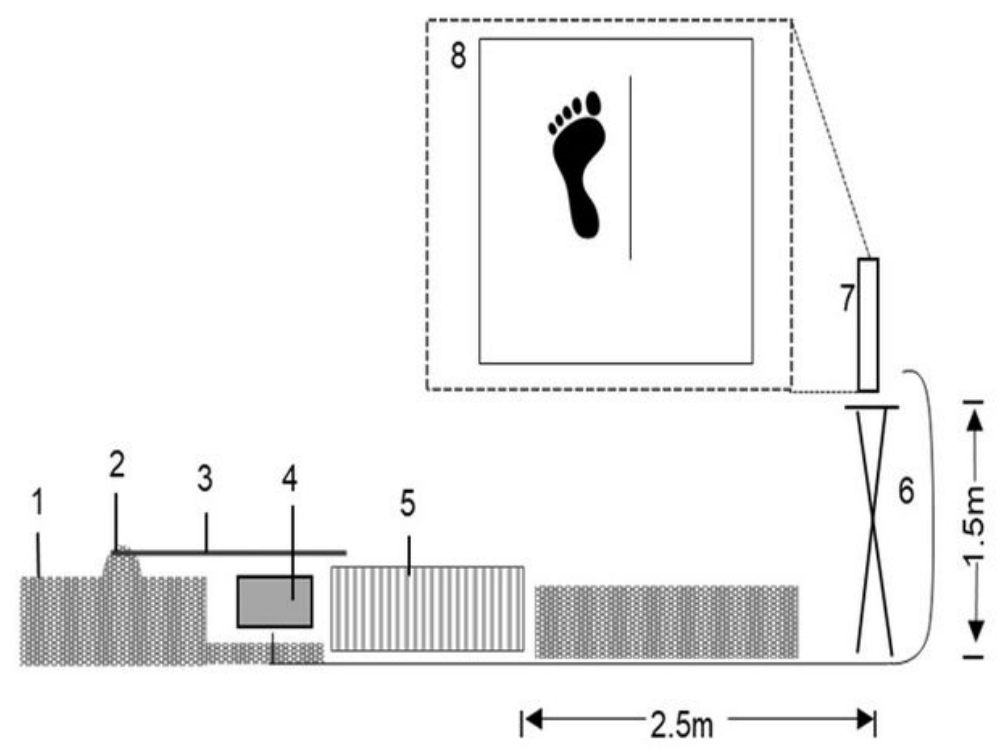


Figure 2

Setup of the jump landing experiment (adapted and modified based on Giesche et. al., 2019). [35]

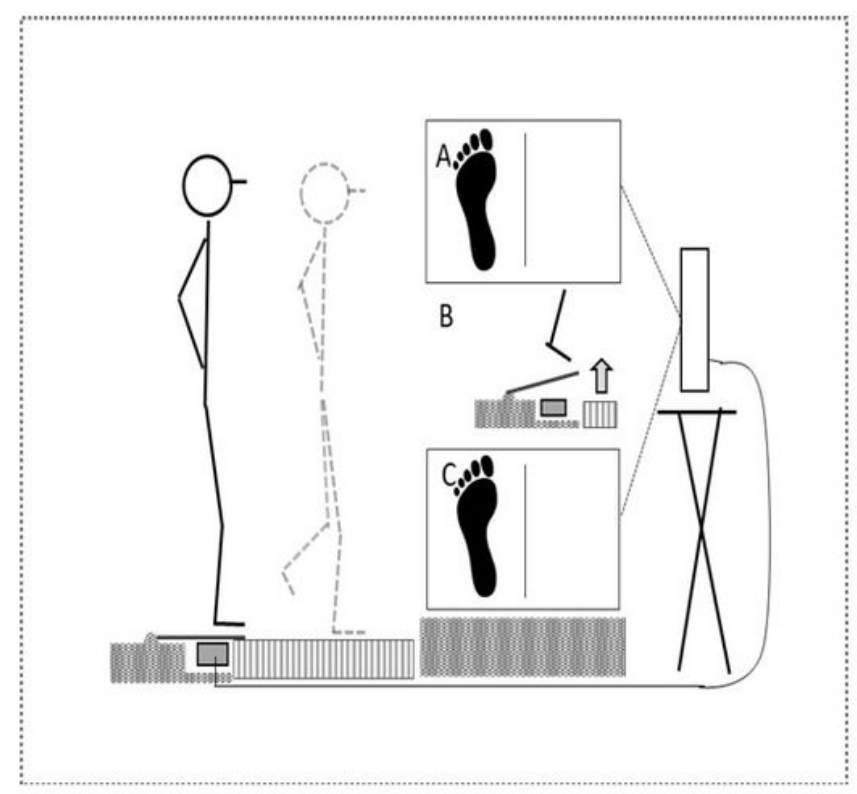

\section{Figure 3}

Anticipated jump landing (adapted and modified based on Giesche et. al., 2019) [35] Participant starts standing in starting position bipedal on plastic panel. A: First Powerpoint-slide (projected) on screen prior to jump initiation (randomised) B: USB footswitch release at take-off to elevate plastic panel and initiate slide change. C: Second Powerpoint-slide displaying expected foot on which to land after jump is initiated 


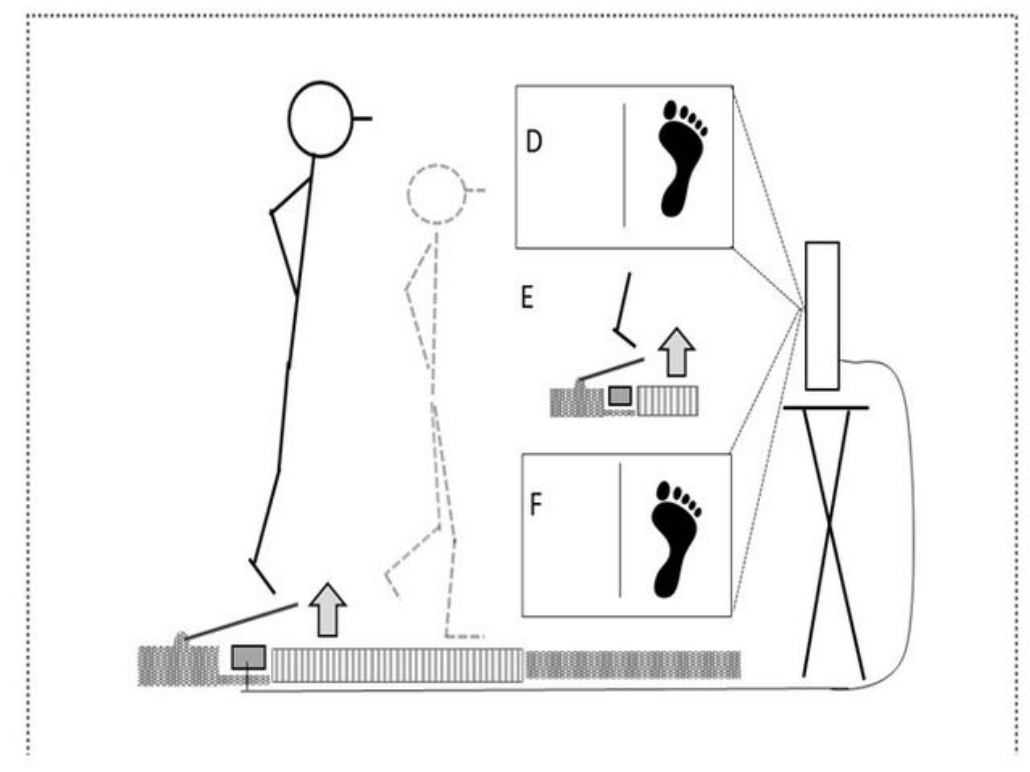

\section{Figure 4}

Unanticipated (consistent) jump landing (adapted and modified based on Giesche et. al., 2019). [35] Participant starts standing in starting position bipedal on plastic panel. D: First Powerpoint-slide (projected) on screen prior to jump initiation (randomised) E: USB footswitch release at take-off to elevate plastic panel and initiate slide change. F: Second Powerpoint-slide displaying expected foot on which to land after jump is initiated 


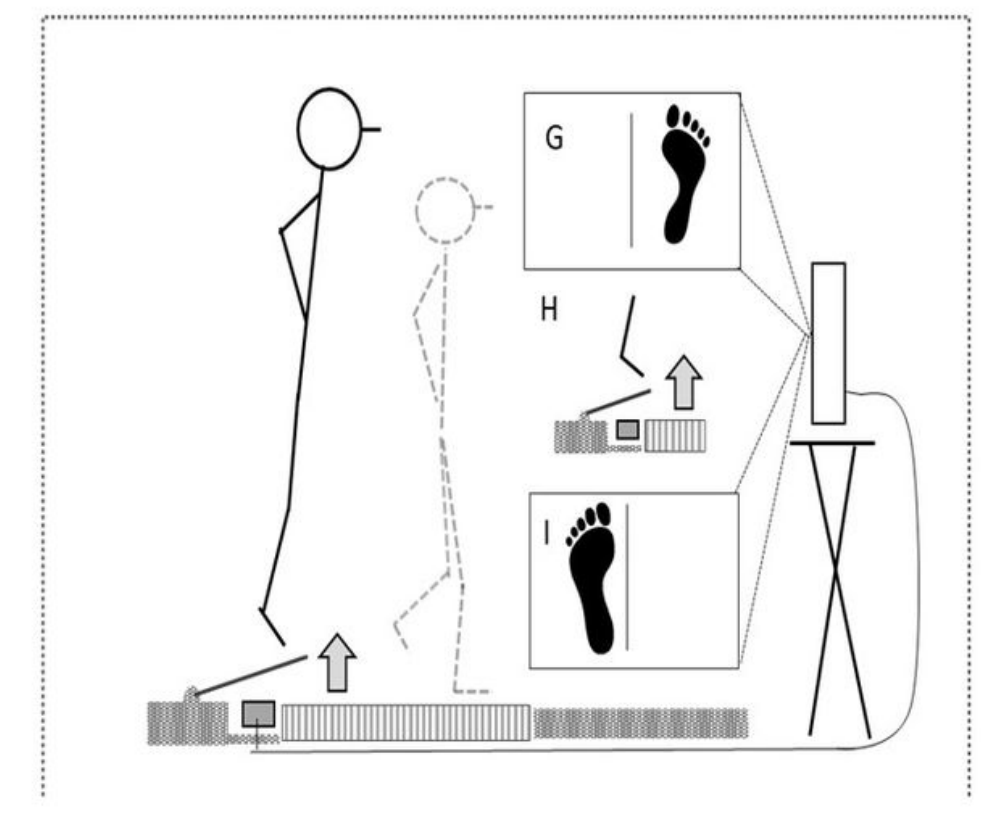

\section{Figure 5}

Unanticipated (inconsistent) jump landing (adapted and modified based on Giesche et. al. 2019). [35] Participant starts standing in starting position bipedal on plastic panel. G: First Powerpoint-slide (projected) on screen prior to jump initiation (randomised) H: USB footswitch release at take-off to elevate plastic panel and initiate slide change. I: Second Powerpoint-slide displaying expected foot on which to land after jump is initiated.

\section{Supplementary Files}

This is a list of supplementary files associated with this preprint. Click to download.

- SPIRITChecklistcompleted.pdf

- Participantsinformationandlnformedconsent.pdf 